\title{
Robust Fractional-Order PID Controller Tuning Based on Bode's Optimal Loop Shaping
}

\author{
Lu Liu $\mathbb{D}^{1}$ and Shuo Zhang $\mathbb{D}^{2}$ \\ ${ }^{1}$ School of Marine Science and Technology, Northwestern Polytechnical University, Xi'an 710072, China \\ ${ }^{2}$ Department of Applied Mathematics, Northwestern Polytechnical University, Xian 710072, China \\ Correspondence should be addressed to Shuo Zhang; zhangshuo1018@nwpu.edu.cn
}

Received 11 February 2018; Accepted 2 April 2018; Published 25 June 2018

Academic Editor: Viet-Thanh Pham

Copyright ( $2018 \mathrm{Lu}$ Liu and Shuo Zhang. This is an open access article distributed under the Creative Commons Attribution License, which permits unrestricted use, distribution, and reproduction in any medium, provided the original work is properly cited.

\begin{abstract}
This paper presents a novel fractional-order PID controller tuning strategy based on Bode's optimal loop shaping which is commonly used for LTI feedback systems. Firstly, the controller parameters are achieved based on flat phase property and Bode's optimal reference model, so that the controlled system is robust to gain variations and can achieve desirable transient performance according to various control requirements. Then, robustness analysis of the controlled system is carried out to support the results. Furthermore, the parameter setting is analyzed to demonstrate the superiority of the proposed controller. At last, some simulation examples are shown to verify the accuracy and usefulness of the proposed control strategy. The proposed fractional-order PID controller does not have any restriction on the controlled plant, so it can be widely applied on both integer-order and fractionalorder systems.
\end{abstract}

\section{Introduction}

Fractional calculus was first mentioned in a letter between Leibniz and L'Hospital about 300 years ago [1]. Not until the last few decades had the related studies been generalized to various fields instead of pure theoretical derivation. A number of studies have revealed the potentialities of fractional calculus, such as engineering [2-4], physics [5], applied mathematics [6, 7], and bioengineering [8]. Among these research fields, fractional-order control technology and fractional-order modeling develop quite fast. Many physical phenomena were proved to be better described by fractionalorder models $[9,10]$. The additional integral and differential orders in fractional-order control algorithms offer more possibilities in enhancing system robustness, stability, and transient performance.

In the control field, PID control is undoubtedly the most widely used control algorithm in industrial applications [11]. So there are abundant reasons to investigate control algorithm which owns the superiorities of both PID controller and fractional calculus. The earliest attempt was made by
Podlubny who proposed the fractional-order PID ( $\mathrm{PI}^{\lambda} \mathrm{D}^{\mu}$ or FOPID) controller [12]. There are two more parameters, that is, integral order $\lambda$ and differential order $\mu$, in FOPID controller compared with traditional PID controller. These extra parameters can serve as additional tuning knobs which may provide more flexibilities in improving system control performance. Petras presented a digital version FOPID controller which was implemented in a DC motor [13]. Fractance circuits and microprocessors were used to achieve the analogue transformation and implementation of the digital FOPID controller. An optimal FOPID controller was designed based on particle swarm optimization and applied to an automatic voltage regulator by Zamani et al. [14] A frequency domain tuning scheme of FOPID controller was proposed by Vinagre et al. [15]. Hamamci investigated an FOPID controller which can stabilize fractional-order systems with time delay [16]. The proposed controller can also be tuned to meet gain and phase margins requirements. The design method of FOPID controller which is suitable to be used in industrial applications was demonstrated by Monje et. al [2]. Moreover, some novel control technologies 
which combined FOPID controller and intelligent control algorithms together have been investigated and were proved to be effective in improving system transient performance $[4,17]$.

Going a step further, there are many FOPID controllers proposed for enhancing system robustness. One of the notable works is CRONE controller with three generations, developed by Oustaloup to deal with different model uncertainties including zero and pole misplacement [18]. Another frequently used method is achieving the flat phase property which is done by making the derivative of the phase to be zero at gain crossover. A PID control scheme based on this property was presented in [19]. Later, the fractional-order PD/PI controllers, which have already been successfully applied in motion control systems, were also designed according to the same isodamping property [20-22]. The extension of this method which can guarantee the system robustness with respect to different parameters variations was studied by Liu et al. [23]. Moreover, Feliu-Batlle et al. presented a Smith predictor based robust fractional-order controller with the application to water distribution [24]. The author has also proposed a robust fractional-order control scheme used in oscillatory systems with large uncertainties in their parameters [25]. A note on FOPID controller design which proposed a robust parameter tuning algorithm using Bode envelopes can be found in [26]. Besides, a robust fractionalorder cascade PD and PI controller was investigated based on fuzzy control technology by Kumar et al. and has been applied to the speed control of a hybrid electric vehicle [27].

In this paper, we focus on the robust FOPID controller design based on Bode's optimal loop shaping which is commonly used for LTI (Linear Time Invariant) feedback systems [28]. Bode's ideal transfer function is served as a reference model in the parameter optimization process. Some related fractional-order controller tuning algorithms were discussed in [29-31]. However, most of them have a specified controlled system type and cannot be widely applied to different kinds of systems. In [29], a PID controller is proposed based on Bode's ideal transfer function, but it only aims at integer-order controlled plants; a similar work can be found in [30], where a robust fractional-order PI controller is designed; however, according to the derivation process, only one certain type of fractional-order controlled plant has been taken into consideration; moreover, the Bode loop shaping with CRONE compensator is discussed in [31], but it only emphasizes the loop shaping accuracy instead of controller tuning algorithm. The application domain of these studies is quite limited. Therefore, a robust fractional-order PID controller tuning algorithm based on Bode's optimal loop shaping which can be widely applied on both integer-order and fractional-order controlled systems is worth exploring in this paper. The controlled system with the proposed controller is robust to gain variations because the phase curve is flat within a certain frequency limit. The effectiveness of the presented robust FOPID controller is demonstrated by the simulation results of several controlled systems including integer-order and fractional-order ones.

The rest of this paper is organized as follows: in Section 2, some preliminaries of fractional calculus are given; detailed analysis of Bode's optimal loop shaping is presented in Section 3; Section 4 illustrates the proposed controller tuning process as well as robustness analysis of the controlled system; simulation results to verify the effectiveness of the proposed algorithm are presented in Section 5; finally, conclusions are made in Section 6.

\section{Preliminaries}

2.1. Fractional-Order Derivative. The fractional-order integral-differential operator ${ }_{t_{0}} D_{t}^{\alpha}$ can be defined as

$$
t_{0} D_{t}^{\alpha}= \begin{cases}\int_{t_{0}}^{t} f(\tau) d \tau^{-\alpha}, & \alpha<0 \\ f(t), & \alpha=0 \\ \frac{d^{\alpha}}{d t^{\alpha}} f(t), & \alpha>0,\end{cases}
$$

where $\alpha \in R$ is the integral or differential order and $t, t_{0}$ are upper and lower limits of the operator, respectively.

So far, there is still no unified definition of fractionalorder derivative. But there have been three generally accepted definitions, that is, Caputo definition, Riemann-Liouville definition, and Grunwald-Letnikov [1]. Each of the three definitions has its own properties. In this paper, we use Caputo definition because it has been extensively used in engineering applications [6,7]. The Caputo derivative of order $\alpha$ for a function $f(t) \in C^{n+1}\left(\left[t_{0},+\infty\right], R\right)$ is defined as [6]

$$
t_{0} D_{t}^{\alpha} f(t)=\frac{1}{\Gamma(n-\alpha)} \int_{t_{0}}^{t} \frac{f^{(n)}(\tau)}{(t-\tau)^{\alpha+1-n}} d \tau
$$

where $n$ is a positive integer, such that $n-1<\alpha \leq n$.

The Laplace transform based on Caputo definition can be expressed as

$$
\mathscr{L}\left\{{ }_{t_{0}} D_{t}^{\alpha} f(t)\right\}=s^{\alpha} F(s)-\sum_{k=0}^{n-1} s^{\alpha-k-1} f^{(k)}\left(t_{0}\right),
$$

where $\mathscr{L}\{\cdot\}$ represents the Laplace transform, $n-1<\alpha<n$, and $s$ is the Laplace transform operator.

2.2. Mittag-Leffler Function. The Mittag-Leffler (ML) function is a generalized form of exponential function. It is widely used in solving fractional-order differential equations [1]. An ML function with two parameters can be written as a power series as [1]

$$
E_{\alpha, \beta}(x)=\sum_{k=0}^{\infty} \frac{x^{k}}{\Gamma(\alpha k+\beta)},
$$

where $\alpha>0, \beta>0$ and $x \in C$. When $\alpha=\beta=1, E_{1,1}(x)=$ $e^{x}$.

When $\beta=1$, the ML function in (4) simplifies to a oneparameter form as

$$
E_{\alpha}(x)=E_{\alpha, 1}(x)=\sum_{k=0}^{\infty} \frac{x^{k}}{\Gamma(\alpha k+1)} .
$$




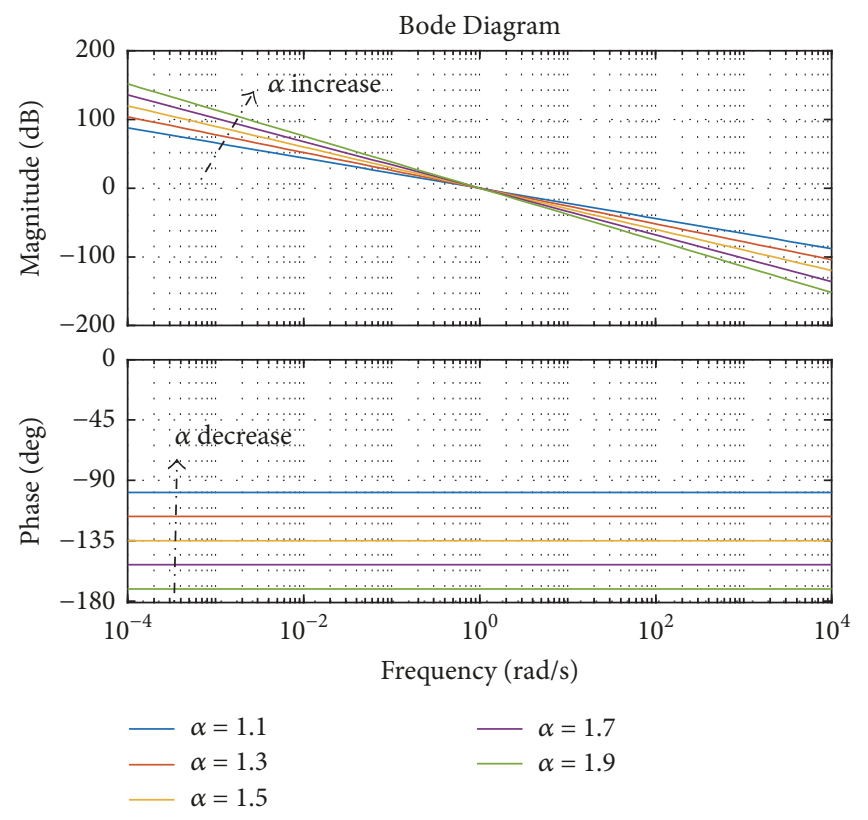

Figure 1: Bode diagram with different $\alpha$.

The Laplace transform of (4) is

$$
\mathscr{L}\left\{t^{\beta-1} E_{\alpha, \beta}\left(-\theta t^{\alpha}\right)\right\}=\frac{s^{\alpha-\beta}}{s^{\alpha}+\theta}, \quad\left(\operatorname{Re}(s)>|\theta|^{1 / \alpha}\right),
$$

where $t \geq 0$ and $\operatorname{Re}(s)$ is the real part of $s$.

\section{Analysis of Bode's Optimal Loop Shaping}

Consider a system whose open-loop transfer function is

$$
F(s)=\frac{A}{s^{\alpha}},
$$

where $\alpha \in R, A=\omega_{c}{ }^{\alpha}$, and $\omega_{c}$ is the crossover frequency of amplitude curve in Bode diagram which satisfies $\lg \left|F\left(j \omega_{c}\right)\right|=$ 0 . System (7) is a typical elementary fractional-order system. The resonance condition of this kind of system is $1<\alpha<2$, which has been proved in [32]. Therefore, we only take $1<$ $\alpha<2$ into consideration in this paper. The Bode diagrams of system (7) with $\alpha=1.1,1.3,1.5,1.7,1.9$ are shown as Figure 1. The amplitude curves are straight lines with the slopes of $-20 \alpha \mathrm{dB} / \mathrm{dec}$, and the corresponding phase curves are horizontal lines with $-\alpha \pi / 2 \mathrm{rad}$. Therefore, the phase margin of system (7) is $(1-\alpha / 2) \pi$. That is to say, the phase margin will not change along with the change of $A$. So system with the open-loop transfer function in (7) is robust to gain variations. This is the optimal loop shaping suggested by Bode in [28].

The closed-loop transfer function corresponding to the open-loop one in (7) is

$$
F(s)=\frac{A}{s^{\alpha}+A}=\frac{1}{T s^{\alpha}+1},
$$

where $1<\alpha<2$ and $T=1 / A=\omega_{c}^{\alpha}$ is the time constant of the system. The closed-loop system in (8) will be

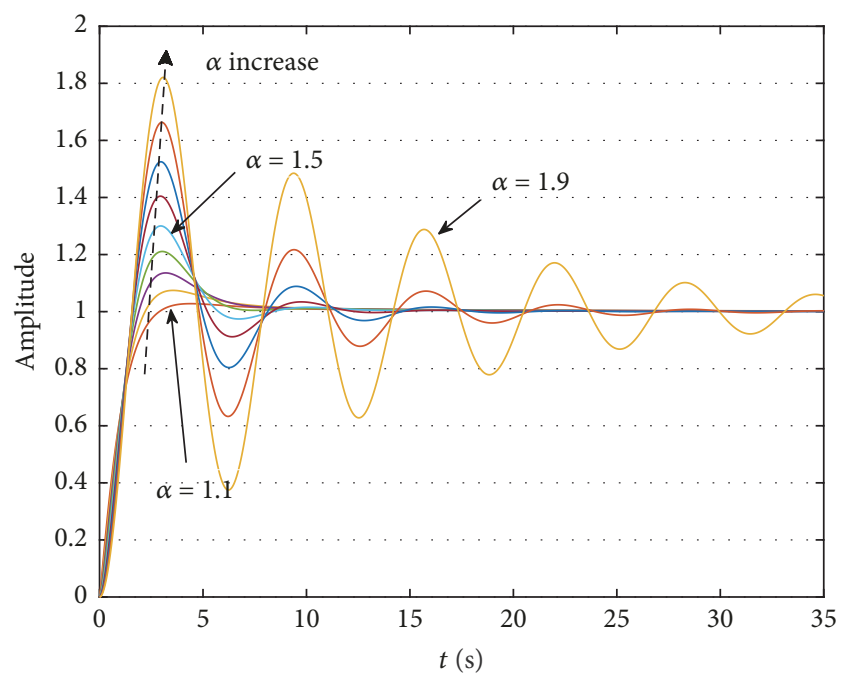

FIGURE 2: Step responses with different $\alpha$.

a reference model in the controller parameters optimization process in next section. Therefore, parameters $\alpha, T$ can be tuned to satisfy different performance requirements.

When a unit step signal is fed into system (8), the time response of the system can be achieved as [1]

$$
\begin{aligned}
y(t) & =L^{-1}\left[\frac{1}{s\left(T s^{\alpha}+1\right)}\right]=1-\sum_{n=0}^{\infty} \frac{\left[-T^{-1} t^{\alpha}\right]^{n}}{\Gamma(\alpha n+1)} \\
& =1-E_{\alpha, 1}\left(-T^{-1} t^{\alpha}\right) .
\end{aligned}
$$

Figure 2 gives the step responses of system (8) with $\alpha=$ $1.1,1.2, \ldots, 1.9$. Some important time response performance indicators are derived as follows. 


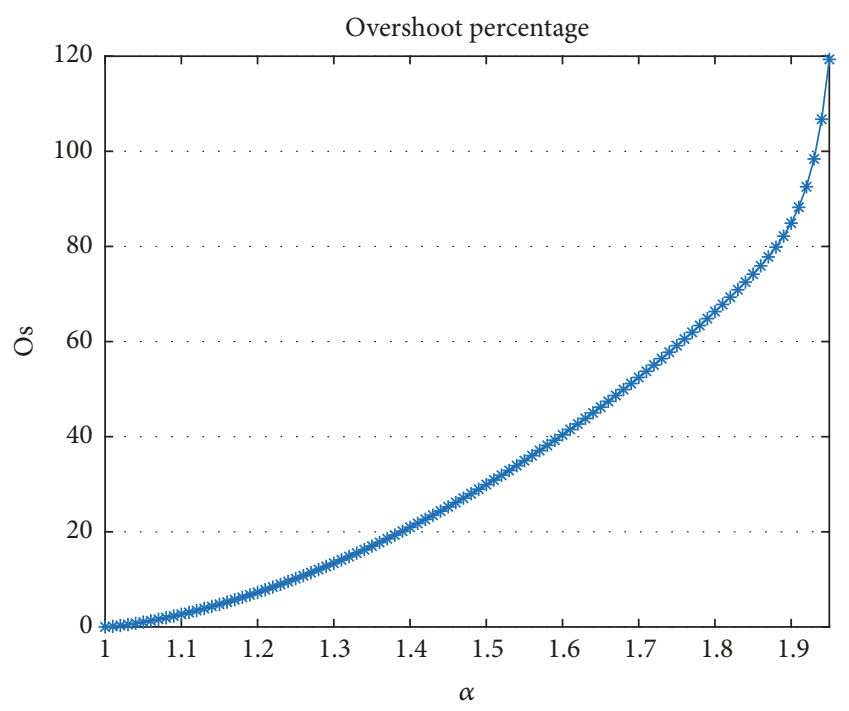

Figure 3: Overshoot percentage with different $\alpha$.

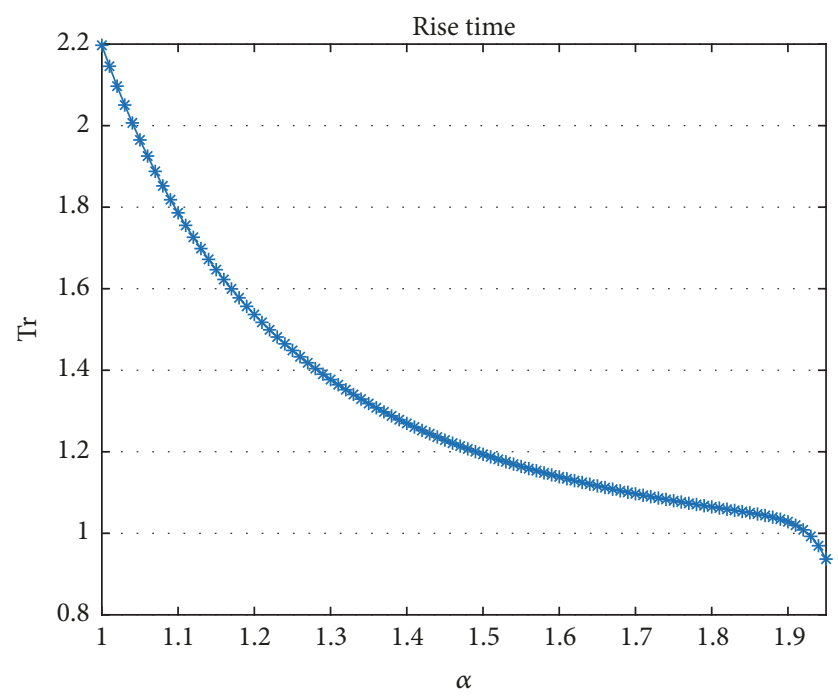

FIGURE 4: Rise time with different $\alpha$.

(1) Overshoot percentage $M_{p}$

$$
M_{p}=\frac{y_{\max }-y_{\text {final }}}{y_{\text {final }}} \times 100 \% .
$$

(2) Rise time $T_{r}$ (the time for the output to rise from $10 \%$ to first time $90 \%$ of the final value).

(3) $2 \%$ or $5 \%$ error band settling time $T_{s}$ (the time for the output to reach and stay within a $2 \%$ or $5 \%$ error band of the final value).

These indicators cannot be achieved explicitly. Therefore, we express the relations between them and parameter $\alpha$ in Figures 3-6. The relationship curves have been approximated numerically into some nonlinear functions in [33]. However, the approximate accuracies may not be satisfactory enough. Hence, we recommend referring to the results we got in the figures. In this way, the controller parameters can be tuned

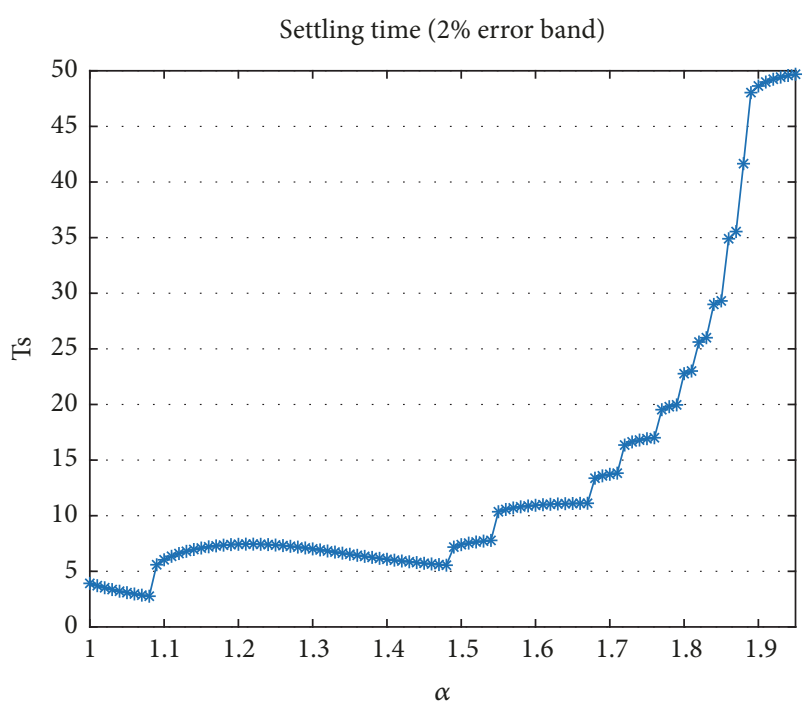

FIGURE 5: Setting time with different $\alpha$ (2\%).

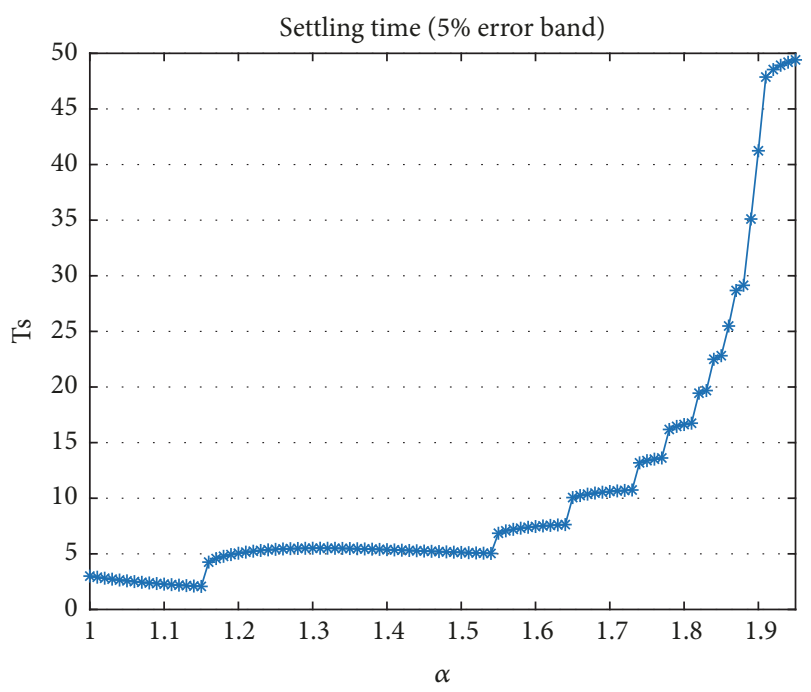

FIGURE 6: Setting time with different $\alpha$ (5\%).

by choosing different $\alpha$ values according to the required time domain indicators.

Moreover, frequency domain response specifications should also be taken into consideration. As it was demonstrated above, the phase margin of system (8) which has correspondence with time domain response overshoot is $(1-\alpha / 2) \pi$. The closed-loop frequency domain responses of system (8) with different $T$ are illustrated in Figure 7. It can be seen from Figure 7 that the bandwidth of the system increases along with the value of $T$. On the other hand, the wider the bandwidth is in a system frequency domain response, the smaller the rise time will be in the corresponding time domain response. Namely, the controller parameters can also be tuned according to frequency domain specifications by changing the values of $\alpha$ and T. Moreover, the curves attenuate fast at high frequencies, which means that the system is not sensitive to high frequency disturbance. 


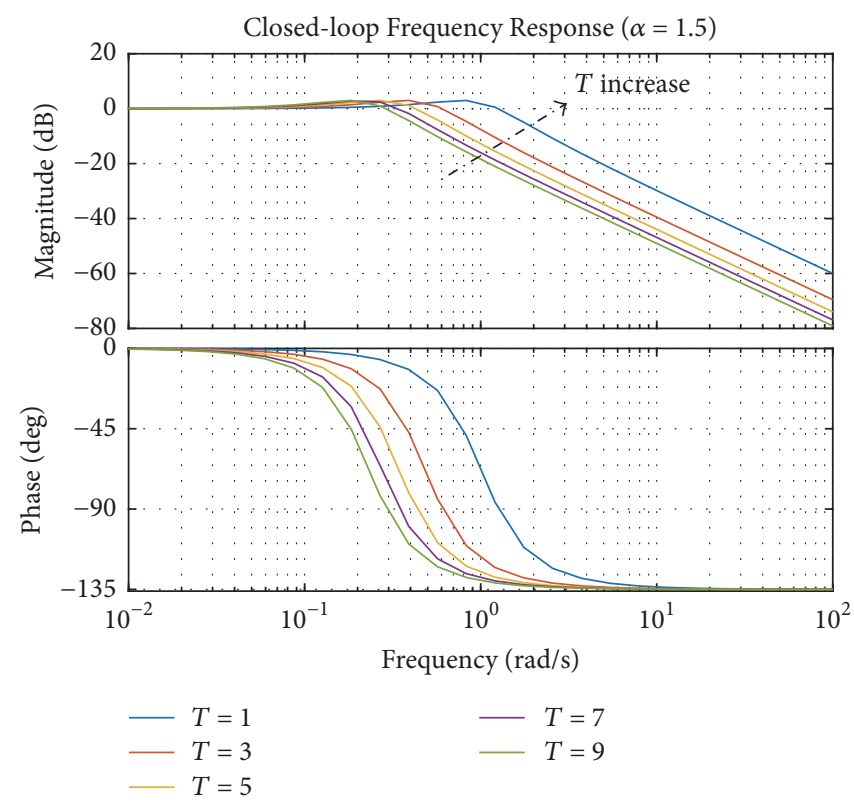

FIGURE 7: Frequency responses with different $T(\alpha=1.5)$.

It should be noted that there are some conflict relations between different design specifications. For instance, parameters chosen according to small rise time will lead to large overshoot. Therefore, wise trade-offs should be made on the selection of reference model.

\section{Controller Tuning}

4.1. Fractional-Order PID Controller. FOPID $\left(\mathrm{PI}^{\lambda} \mathrm{D}^{\mu}\right)$ controller is an extension of conventional PID controller with extra two parameters, that is, integral order $\lambda$ and differential order $\mu$ [12]. It can be expressed in frequency domain as

$$
G_{c}(s)=K_{p}+\frac{K_{i}}{s^{\lambda}}+K_{d} s^{u}, \quad(0<\lambda, \mu<2)
$$

where $K_{p}, K_{i}, K_{d}$ are proportional, integral, and differential coefficients, respectively. When $\lambda=\mu=1$, FOPID controller is equal to conventional PID controller. Figure 8 gives the comparison of controller parameters tuning domains between FOPID and PID controllers in geometrical perspective. The two extra parameters make the controller parameter tuning domain extend from a few points into a partial quarter plane. Meanwhile, the slope of amplitude curve in open-loop system frequency response at crossover frequency updates from $-20 \mathrm{~dB} / \mathrm{dec}$ into $-20 \lambda \mathrm{dB} / \mathrm{dec}$. These provide more possibilities in improving control system robustness as well as transient performance, especially for fractional-order systems. However, the regulating process may become more complicated at the same time. The detailed analysis of the influence of $\lambda, \mu$ on control system performance can be found in [4].

4.2. Fractional Operator Realization. The fractional operators in (11) can be realized by several approaches [1]. In this paper, taking system (7) into consideration, we choose the

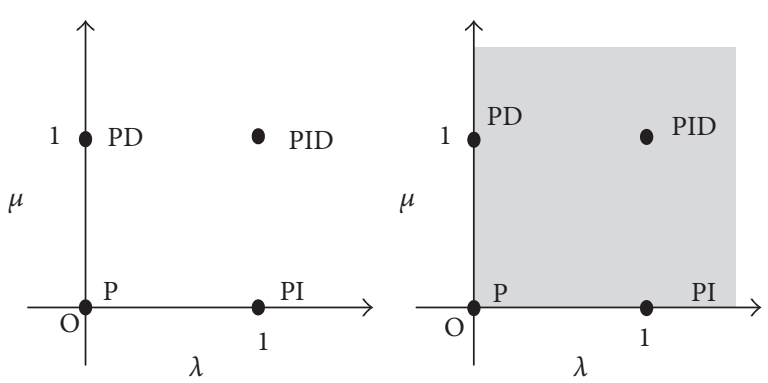

FIGURE 8: Comparison of PID and FOPID controllers.

Oustaloup approximation method which is widely used in this field [34]. A generalized fractional operator can be expressed as

$$
H(s)=\left(\frac{s}{\omega_{i}}\right)^{\alpha}, \quad \alpha \in R^{+} .
$$

Consider the expected approximate frequency range as $\left(\omega_{a}, \omega_{b}\right)$; the operator $s / \omega_{i}$ can be substituted by

$$
K_{0} \frac{1+s / \omega_{s}}{1+s / \omega_{l}}
$$

where $K_{0}=\omega_{s} / \omega_{i}=\omega_{i} / \omega_{l}, \omega_{s}<\omega_{a}, \omega_{l}>\omega_{b}$. Therefore, (12) can be updated as

$$
H(s)=K\left(\frac{1+s / \omega_{s}}{1+s / \omega_{l}}\right)^{\alpha},
$$

where $C=C_{0}{ }^{\alpha}$. Transform the transfer function above into the zero-pole form; it is obtained that

$$
H(s)=\lim _{n \rightarrow \infty} \hat{H}(s),
$$




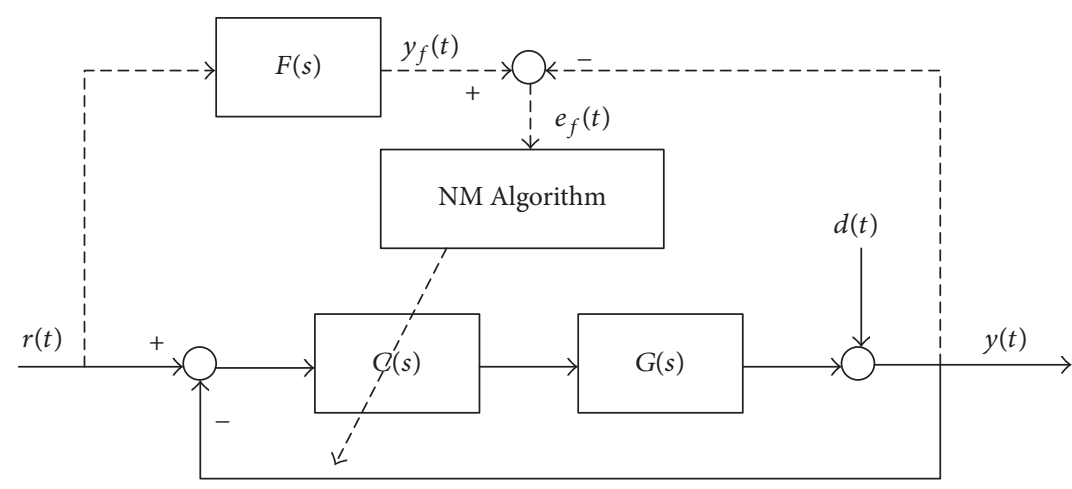

FIgURE 9: Tuning structure.

where

$$
\begin{aligned}
\hat{H}(s) & =\left(\frac{\omega_{i}}{\omega_{l}}\right)^{\alpha} \prod_{k=-N}^{N} \frac{1+s / \omega_{k}}{1+s / \omega_{k}^{\prime}}, \\
\omega_{k} & =\omega_{s}\left(\frac{\omega_{l}}{\omega_{s}}\right)^{((1 / 2)(1-\alpha)+k+N) /(2 N+1)}, \\
\omega_{k}^{\prime} & =\omega_{s}\left(\frac{\omega_{l}}{\omega_{s}}\right)^{((1 / 2)(1+\alpha)+k+N) /(2 N+1)} .
\end{aligned}
$$

In this paper, we use the 7 th approximation with interested frequency range $\left(10^{-3}, 10^{3}\right)$.

4.3. Parameter Optimization. Normally, controller parameter optimization is proceeded based on the objective function which includes error between reference signal and actual output. However, this tuning method can only randomly get satisfactory control performance. It may make the rise time or overshoot of the controlled system quite small, but it cannot guarantee meeting any specific requirement. Besides, large times of trial and error are needed in the tuning process. In this paper, the Bode's optimal loop shaping transfer function is used as a nominal reference model in the optimization process to help the controlled system satisfy different control performance requirements. The tuning structure of the controlled system is represented in Figure 9, where $r(t), y(t), d(t)$ are reference signal, actual output signal, and disturbance signal, $e_{f}(t), y_{f}(t)$ are nominal error signal and desire output signal which satisfy $e_{f}(t)=y_{f}(t)-y(t)$, and $C(s), G(s), F(s)$ are controller, controlled plant, and nominal reference model, respectively. In this way, the robustness and other transient performance requirements can be improved by tuning the controller parameters as well as the parameters of the nominal reference model $F(s)$ which was discussed in Section 3. The ITAE (Integral Time Absolute Error) indicator is chosen as the objective function in this paper. Therefore, according to Figure 9, the objective function can be expressed as

$$
J=\int_{0}^{\infty} t\left|e_{f}(t)\right| d t=\int_{0}^{\infty} t\left|y_{f}(t)-y(t)\right| d t
$$

After getting the objective function, the Nelder-Mead (NM) simplex search algorithm is used to optimize the controller parameters according to the value of the objective function [35]. The fundamental principle of NM algorithm is constructing a rough search direction according to the initial parameter values first and updating the search direction constantly according to the objective function value until the optimal result is achieved. NM algorithm is widely applied in optimization processes, but the initial values influence the optimization result a lot. Therefore, suitable initial values should be selected before optimization.

4.4. Robustness Analysis. In this subsection, the robustness of a typical fractional-order system controlled by the proposed controller is analyzed. Consider a fractional-order transfer function described by

$$
P(s)=\frac{k}{a_{1} s^{\alpha_{1}}+a_{2} s^{\alpha_{2}}+1},
$$

where $k>0$ is the system gain, $a_{1}$ and $a_{2}$ are positive constant coefficients, and the fractional-orders satisfy $1<\alpha_{1} \leq 2, \quad 0<$ $\alpha_{2} \leq 1$.

Next we consider the corresponding FOPID controller designed as (11), where the fractional-orders are chosen as $\lambda=\alpha_{2}$ and $\mu=\alpha_{1}-\alpha_{2}$ and the controller parameters are set as $k_{p}=a_{2} / b, k_{i}=1 / b$, and $k_{d}=a_{2} / b$. The constant $b$ satisfies $b=k \omega_{c}^{-\alpha_{2}}$, and $\omega_{c}$ represents the gain crossover frequency of the open-loop transfer function $L(s)=P(s) G_{c}(s)$.

Based on (11) and (18), the open-loop transfer function can be obtained as

$$
L(s)=\frac{k}{b s^{\alpha_{2}}}=\left(\frac{\omega_{c}}{s}\right)^{\alpha_{2}} .
$$

Then we analyze the robustness of the controlled system with designed FOPID controller by employing the small gain theorem which has been widely used in FOPID control [3638]. According to the small gain theorem, the robust stability condition can be represented by

$$
\delta_{p}(\omega)<V\left(\omega, \alpha_{2}\right) \triangleq \frac{|1+P(\omega i) C(\omega i)|}{|P(\omega i) C(\omega i)|},
$$

for $\forall \omega>0$,

where $\delta_{p}(\omega)$ denotes the multiplicative norm-bound uncertainty of transfer function $P(s)$. Thus, larger $V\left(\omega, \alpha_{2}\right)$ means 
TABLE 1: Controller parameters of $P_{1}(s)$.

\begin{tabular}{lccccc}
\hline$\phi_{m}=45^{\circ}$ & $K_{p}$ & $K_{i}$ & $K_{d}$ & $\lambda$ & $\mu$ \\
\hline$\omega_{c}=0.5$ & 0.4576 & 4.8570 & 0.0051 & 1.3814 & 1.8969 \\
$\omega_{c}=1.0$ & 0.2174 & 1.8775 & 0.0125 & 1.4226 & 0.0653 \\
$\omega_{c}=1.5$ & 0.1494 & 1.0816 & 0.0032 & 1.4226 & 2.1905 \\
\hline$\phi_{m}=60^{\circ}$ & $K_{p}$ & $K_{i}$ & $K_{d}$ & $\lambda$ & $\mu$ \\
\hline$\omega_{c}=0.5$ & 0.4197 & 4.7618 & 0.0907 & 1.2399 & 0.0468 \\
$\omega_{c}=1.0$ & 0.0649 & 1.8576 & 0.1888 & 1.2794 & 0.0023 \\
$\omega_{c}=1.5$ & 0.1719 & 1.0697 & 0.0328 & 1.2957 & 1.2878 \\
\hline$\phi_{m}=90^{\circ}$ & $K_{p}$ & $K_{i}$ & $K_{d}$ & 0.9969 & $\mu$ \\
\hline$\omega_{c}=0.5$ & 0.9640 & 4.3890 & 0.0063 & 0.9976 & 1.1411 \\
$\omega_{c}=1.0$ & 0.3930 & 1.7269 & 0.0150 & 0.9981 & 2.1141 \\
$\omega_{c}=1.5$ & 0.2327 & 1.003 & 0.0080 &
\end{tabular}

that bigger modeling uncertainty may occur without breaking the robust stability of the controlled system.

Due to (11), (18), and (20), we achieve $V\left(\omega, \alpha_{2}\right)$ as

$$
\begin{gathered}
V\left(\omega, \alpha_{2}\right)=\frac{\left|1+\left(\omega_{c} / \omega i\right)^{\alpha_{2}}\right|}{\left|\left(\omega_{c} / \omega i\right)^{\alpha_{2}}\right|}=\frac{\left|1+i^{\left(-\alpha_{2}\right)}\left(\omega_{c} / \omega\right)^{\alpha_{2}}\right|}{\left(\omega_{c} / \omega\right)^{\alpha_{2}}} \\
=\left(\left(\frac{\omega}{\omega_{c}}\right)^{2 \alpha_{2}}+2\left(\frac{\omega}{\omega_{c}}\right)^{\alpha_{2}} \cos \left(\frac{\alpha_{2} \pi}{2}\right)+1\right)^{1 / 2} .
\end{gathered}
$$

Besides, we calculate the derivatives of $V\left(\omega, \alpha_{2}\right)$ with respect to $\alpha_{2}$ and obtain

$$
\begin{aligned}
& \frac{d V\left(\omega, \alpha_{2}\right)}{d \alpha_{2}} \\
& =\frac{\ln \left(\omega / \omega_{c}\right)\left[\left(\omega / \omega_{c}\right)^{\alpha_{2}}+\cos \left(\alpha_{2} \pi / 2\right)\right]-(\pi / 2) \sin \left(\alpha_{2} \pi / 2\right)}{\sqrt{\left(1+2\left(\omega_{c} / \omega\right)^{\alpha_{2}} \cos \left(\alpha_{2} \pi / 2\right)+\left(\omega_{c} / \omega\right)^{2 \alpha_{2}}\right)}} .
\end{aligned}
$$

It is obvious that $d V\left(\omega, \alpha_{2}\right) / d \alpha_{2}<0$ when $\omega \leq \omega_{c}$ and $0<\alpha_{2} \leq 1$. Thus, $V\left(\omega, \alpha_{2}\right)$ is monotone decreasing with respect to $\alpha_{2} \in(0,1]$. In other words, for the same control system, FOPID controller owns better robust performance than PID controller in the low or even medium frequency ranges, which is established by the gain crossover frequency $\omega \leq \omega_{c}$, and has no connection with the fractional-order $\alpha_{2}$. In the simulation part, the obtained robust analysis result will be verified by some numerical examples.

\section{Simulation}

In this section, we take three typical kinds of systems, including fractional-order system and integer-order system, into consideration to illustrate the effectiveness of the proposed control strategy. Firstly, consider a fractional-order system whose transfer function is similar to the widely used firstorder system [30]:

$$
P_{1}(s)=\frac{0.5856}{0.2318 s^{0.985}+1} .
$$

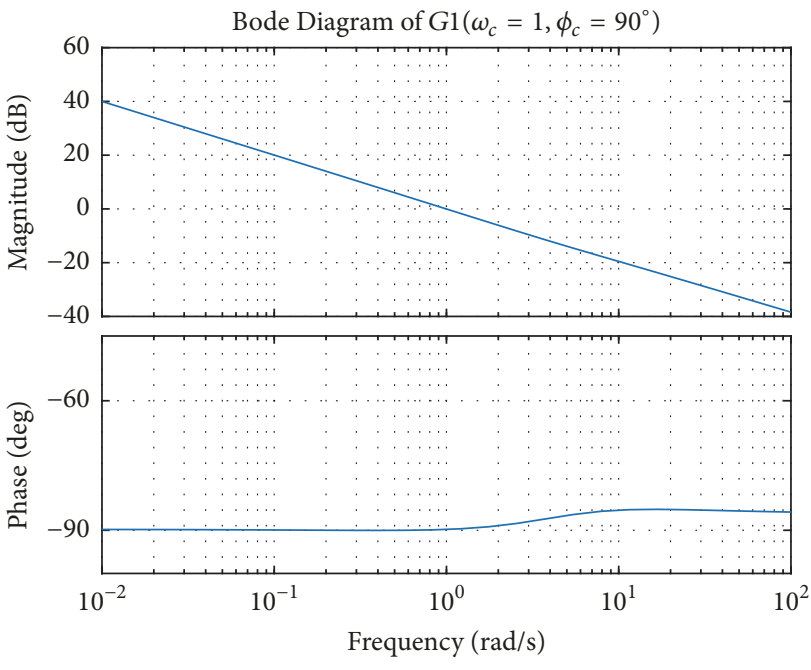

Figure 10: Bode diagram of $P_{1}(s)$.

The reference parameters in (8) and controller parameters in (11) tuned by the proposed algorithm are shown in Table 1. The Bode diagram of $P_{1}(s)$ controlled by the proposed controller with reference model parameters $\omega_{c}=1, \phi_{m}=90^{\circ}$ is depicted in Figure 10. It can be seen that the phase curve at crossover frequency is almost flat and the phase margin is $\pi / 2$ which satisfies the controller design requirements. The step responses of $P_{1}(s)$ controlled by FOPID controllers tuned with the fixed phase margin $\phi_{m}=60^{\circ}$ and different crossover frequencies $\omega_{c}=0.5, \omega_{c}=1, \omega_{c}=1.5$ are shown in Figure 11. Correspondingly, Figure 12 demonstrates the step responses of $P_{1}(s)$ controlled by FOPID controllers tuned with the fixed crossover frequency $\omega_{c}=1$ and varying phase margins $\phi_{m}=45^{\circ}, \phi_{m}=60^{\circ}, \phi_{m}=90^{\circ}$. The unchanged overshoot and different settling times in Figure 11 and the similar settling time and different overshoots in Figure 12 illustrate the impact of reference model with varying parameters. Therefore, different control requirements can be fulfilled by varying reference model parameters $\omega_{c}$, $\phi$ and controller parameters. In order to check the robustness of the controlled 


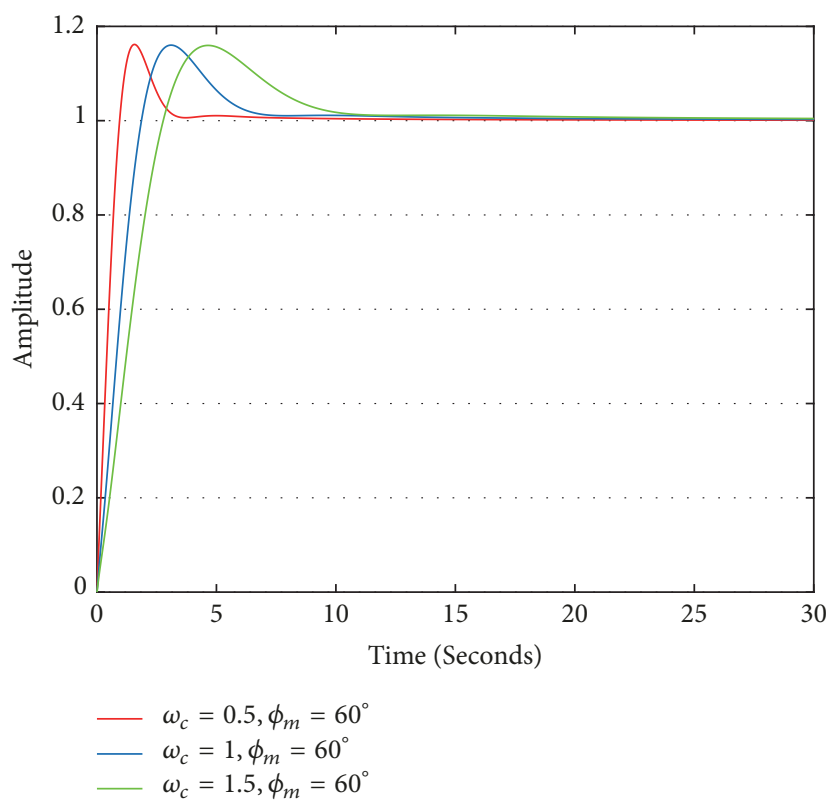

FIGURE 11: Step responses of $P_{1}(s)$ with different $\omega_{c}$.

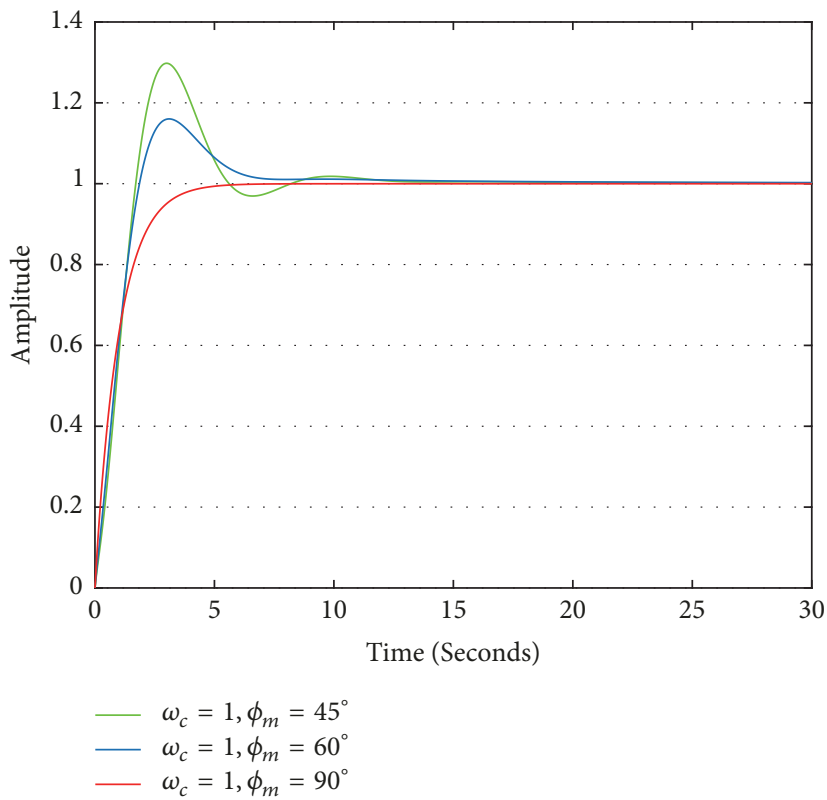

FIGURE 12: Step responses of $P_{1}(s)$ with different $\phi_{m}$.

system, step responses comparisons of systems tuned by different reference parameters under $\pm 30 \%$ gain variations are demonstrated in Figures 13 and 14, respectively. The overshoots of the step responses in each subfigures remain almost the same. These phenomena show that system $P_{1}(s)$ controlled by the proposed controller is robust to high amplitude gain variations. The step response comparison of $P_{1}(s)$ controlled by the proposed FOPID controller and the FOPID controller used in [30] is shown in Figure 15. The performance of system controlled by the proposed controller outperforms the other one with better robustness, lower overshoot, and smaller settling time. Figure 16 illustrates the ITAE indices values of $P_{1}(s)$ with different tuning parameters. All the indices are small enough to meet the optimization requirements.

Then, consider a fractional-order system [1] which is relatively complicated as

$$
P_{2}(s)=\frac{1}{s^{2.6}+3.3 s^{1.5}+2.9 s^{1.3}+3.32 s^{0.9}+1} .
$$




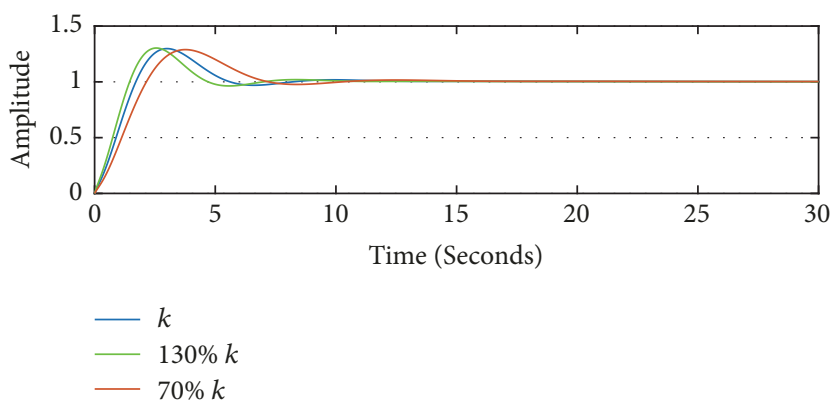

(a) $\omega_{c}=1, \phi_{m}=45^{\circ}$

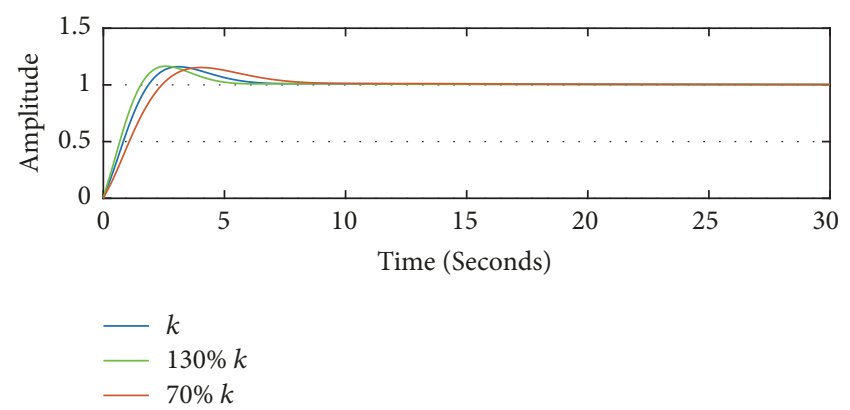

(b) $\omega_{c}=1, \phi_{m}=60^{\circ}$

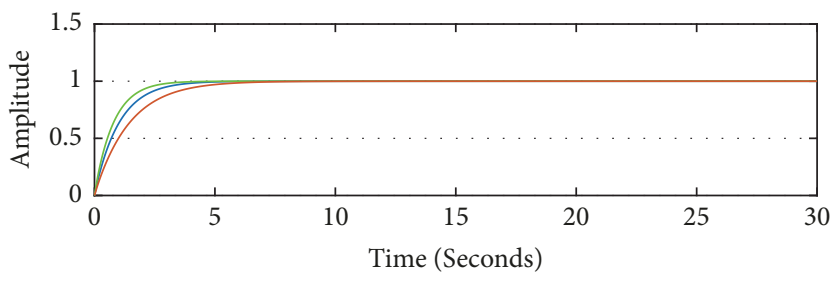

$\begin{array}{ll}k \\ - & 130 \% k\end{array}$

$-70 \% k$

(c) $\omega_{c}=1, \phi_{m}=90^{\circ}$

FIGURE 13: Step responses of $P_{1}(s)$ with different $\phi_{m}$.
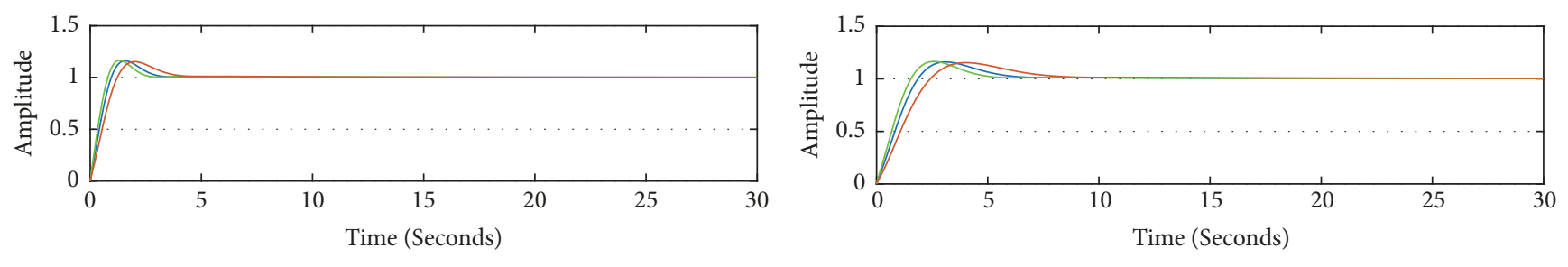

$-\quad k$
$-130 \% k$
$-70 \% k$

$-k$

$-130 \% k$

$-70 \% k$

(a) $\omega_{c}=0.5, \phi_{m}=60^{\circ}$

(b) $\omega_{c}=1, \phi_{m}=60^{\circ}$

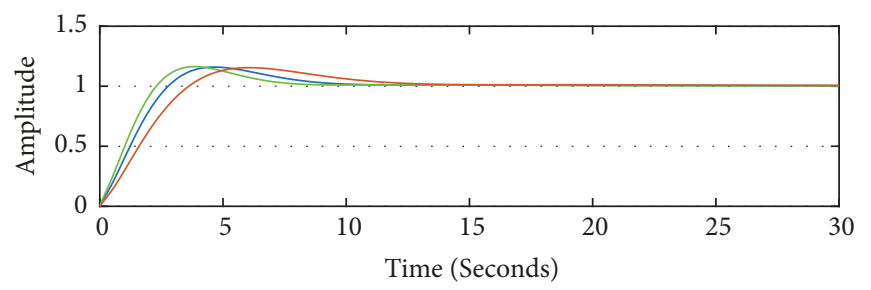

$-k$
$-130 \% k$
$-70 \% k$

(c) $\omega_{c}=1.5, \phi_{m}=60^{\circ}$

FIGURE 14: Step responses of $P_{1}(s)$ with different $\omega_{c}$.

The reference parameters and the obtained the controller parameters are shown in Table 2. Figures 17 and 18 demonstrate the step responses of $P_{2}(s)$ controlled by the proposed controllers tuning with different $\omega_{c}$ and $\phi_{m}$. Similarly, the results show that different control performance can be satisfied by varying reference model parameters. The ITAE indices of $P_{2}(s)$ in Figure 19 are also small enough for optimization. 


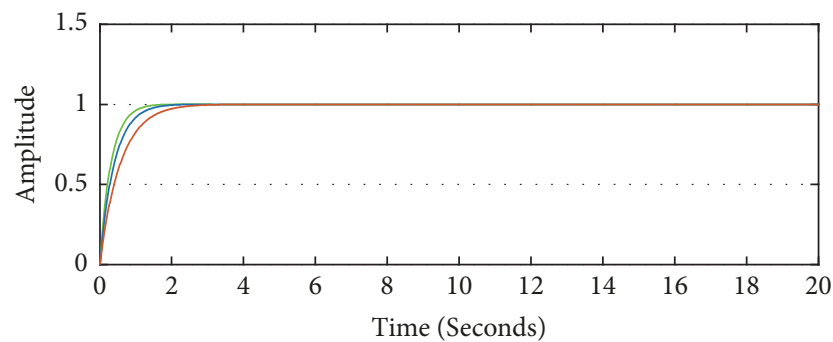

$\begin{array}{ll}- & k \\ - & 130 \% k \\ - & 70 \% k\end{array}$

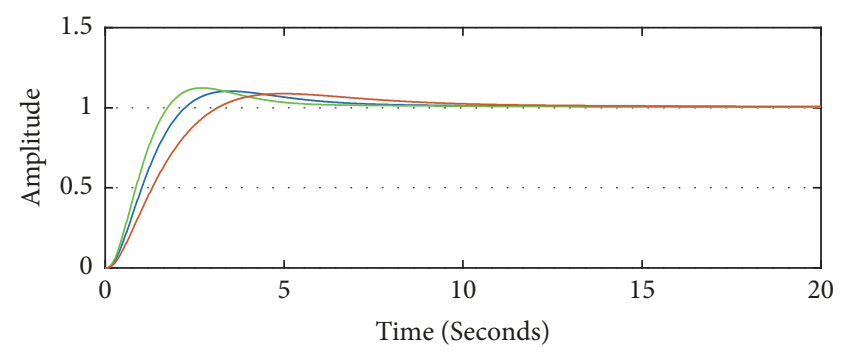

$-k$

$130 \% k$

$70 \% k$

(a)

(b)

FIGURE 15: Step responses of $P_{1}(s)$ with different FOPID controllers ((a) FOPID controller proposed in this paper, (b) FOPID controller in [30]).

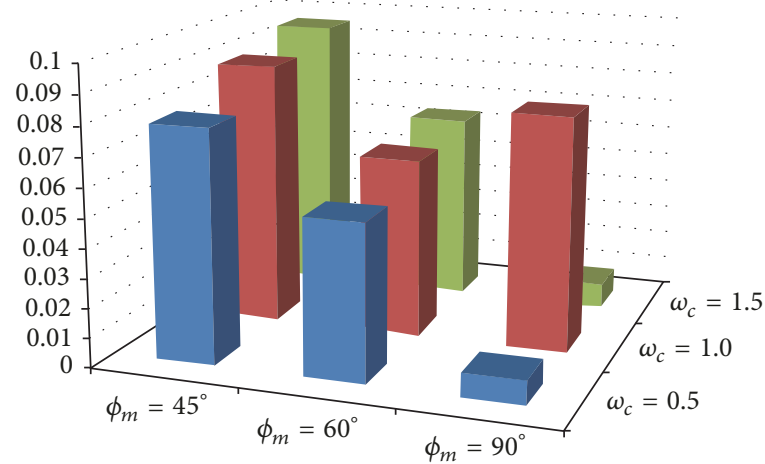

Figure 16: ITAE indices values of $P_{1}(s)$.

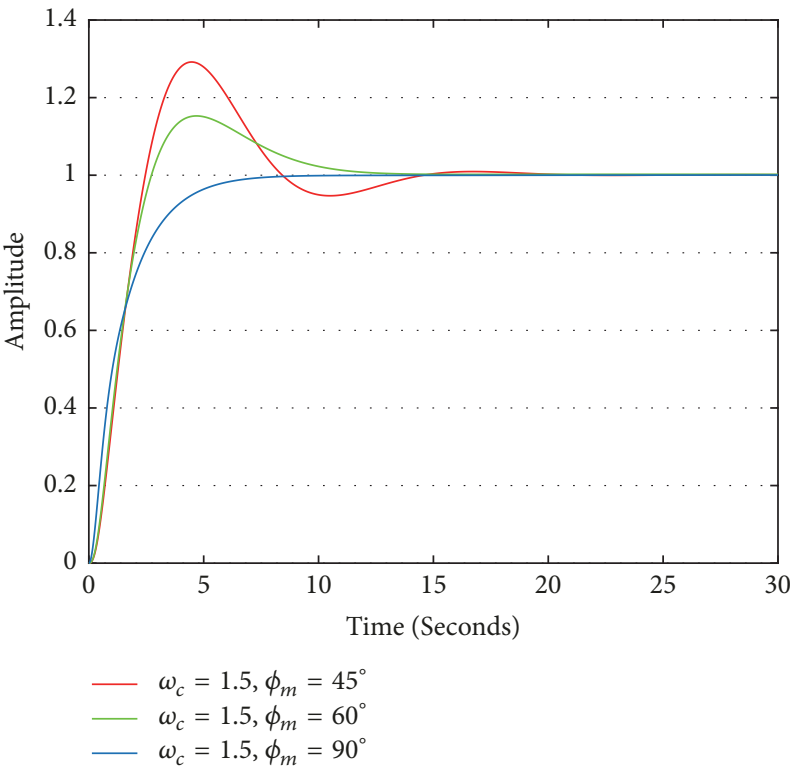

FIGURE 17: Step response of $P_{2}(s)$ with the same $\omega_{c}$. 


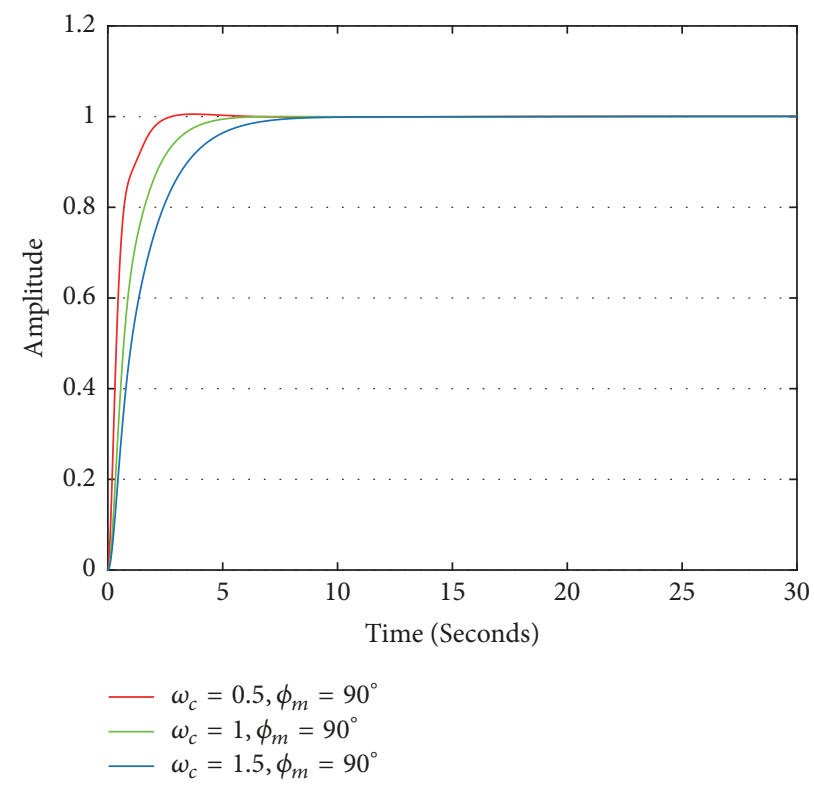

Figure 18: Step response of $P_{2}(s)$ with the same $\phi_{m}$.

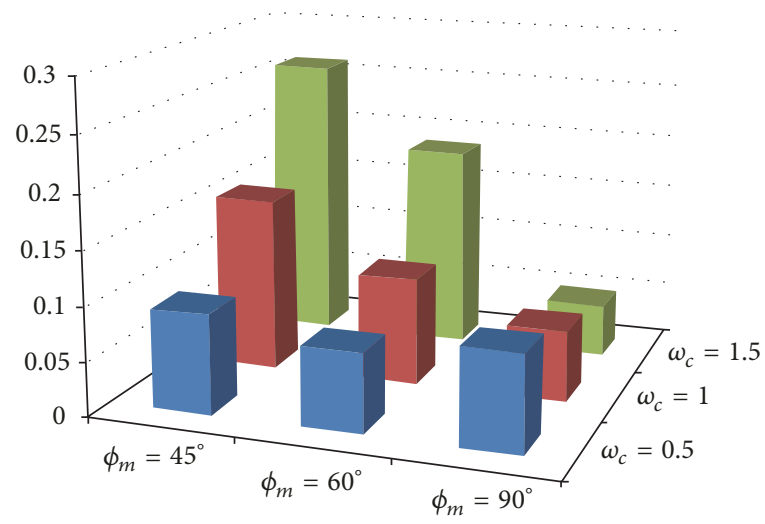

FIGURE 19: ITAE indices values of $P_{2}(s)$.

TABLE 2: Controller parameters of $P_{2}(s)$.

\begin{tabular}{|c|c|c|c|c|c|}
\hline$\phi_{m}=45^{\circ}$ & $K_{p}$ & $K_{i}$ & $K_{d}$ & $\lambda$ & $\mu$ \\
\hline$\omega_{c}=0.5$ & 18.3282 & 9.9945 & 1.5199 & 0.9742 & 1.3785 \\
\hline$\omega_{c}=1.0$ & 7.9000 & 3.4213 & 0.5194 & 1.0581 & 1.6645 \\
\hline$\omega_{c}=1.5$ & 3.4053 & 1.7451 & 0.8343 & 1.0783 & 0.0200 \\
\hline$\phi_{m}=60^{\circ}$ & $K_{p}$ & $K_{i}$ & $K_{d}$ & $\lambda$ & $\mu$ \\
\hline$\omega_{c}=0.5$ & 19.9228 & 4.6069 & 2.0377 & 1.0861 & 1.3092 \\
\hline$\omega_{c}=1.0$ & 8.7852 & 1.9719 & 0.9068 & 1.1120 & 1.4516 \\
\hline$\omega_{c}=1.5$ & 2.6270 & 1.0729 & 2.2338 & 1.1239 & 0.0068 \\
\hline$\phi_{m}=90^{\circ}$ & $K_{p}$ & $K_{i}$ & $K_{d}$ & $\lambda$ & $\mu$ \\
\hline$\omega_{c}=0.5$ & 13.1618 & 1.6627 & 6.6312 & 1.0474 & 0.8915 \\
\hline$\omega_{c}=1.0$ & 5.8327 & 0.9930 & 3.7246 & 1.0143 & 0.6984 \\
\hline$\omega_{c}=1.5$ & 3.7370 & 0.6459 & 2.5961 & 1.0057 & 0.6438 \\
\hline
\end{tabular}


TABLE 3: Controller parameters of $P_{3}(s)$.

\begin{tabular}{|c|c|c|c|c|c|}
\hline$\phi_{m}=45^{\circ}$ & $K_{p}$ & $K_{i}$ & $K_{d}$ & $\lambda$ & $\mu$ \\
\hline$\omega_{c}=0.5$ & 2.4905 & 4.3983 & 0.0580 & 1.2311 & 1.5110 \\
\hline$\omega_{c}=1.0$ & 0.9355 & 1.4819 & 0.0018 & 1.2690 & 0.6910 \\
\hline$\omega_{c}=1.5$ & 0.6170 & 0.7823 & 0.0267 & 1.3210 & 2.0922 \\
\hline$\phi_{m}=60^{\circ}$ & $K_{p}$ & $K_{i}$ & $K_{d}$ & $\lambda$ & $\mu$ \\
\hline$\omega_{c}=0.5$ & 2.7170 & 3.4615 & 0.1476 & 1.1772 & 1.3201 \\
\hline$\omega_{c}=1.0$ & 1.1504 & 1.3321 & 0.0444 & 1.2078 & 1.5853 \\
\hline$\omega_{c}=1.5$ & 0.7038 & 0.7593 & 0.0268 & 1.2262 & 1.7678 \\
\hline$\phi_{m}=90^{\circ}$ & $K_{p}$ & $K_{i}$ & $K_{d}$ & $\lambda$ & $\mu$ \\
\hline$\omega_{c}=0.5$ & 2.4535 & 1.9657 & 0.4621 & 1.0005 & 1.0355 \\
\hline$\omega_{c}=1.0$ & 1.2374 & 0.9942 & 0.2407 & 1.0002 & 1.0212 \\
\hline$\omega_{c}=1.5$ & 0.8273 & 0.6647 & 0.1628 & 1.0001 & 1.0157 \\
\hline
\end{tabular}

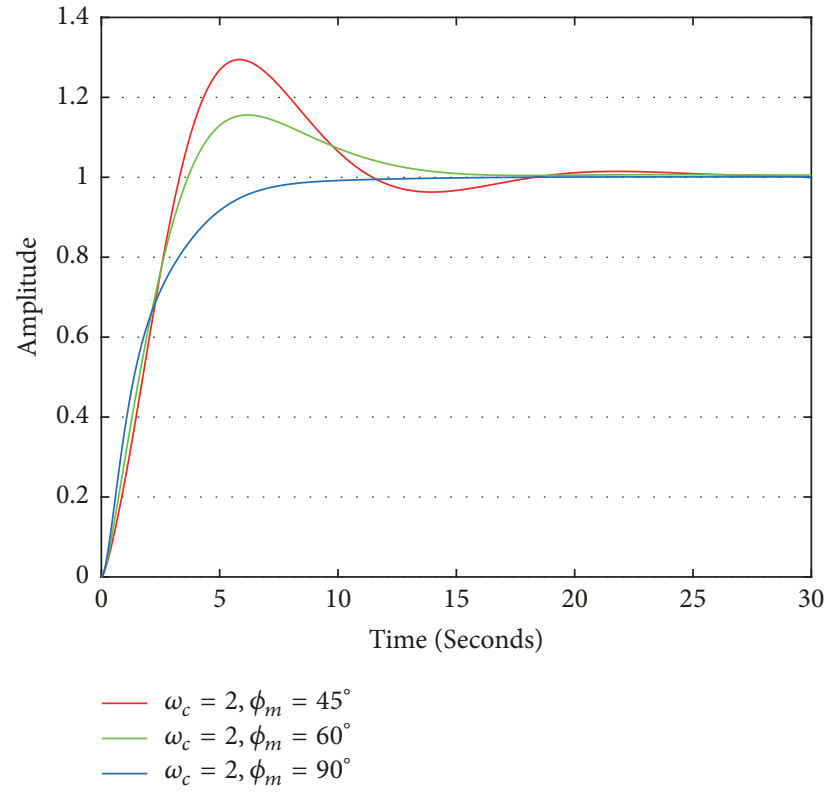

Figure 20: Step response of $P_{3}(s)$ with the same $\omega_{c}$.

Finally, take an integer-order system [31], whose transfer function is shown as below, into consideration:

$$
P_{3}(s)=\frac{1}{(s+1)(0.2 s+1)(0.04 s+1)(0.008 s+1)} .
$$

Table 3 shows the tuned controller and reference model parameters of $P_{3}(s)$. Step responses of $P_{3}(s)$ controlled by different controllers in Table 3 are depicted in Figures 20 and 21. It is shown that the step responses of integer-order system can also be shaped by the proposed FOPID controller according to different design requirements. The ITAE indices of $P_{3}(s)$ in Figure 22 also satisfy the optimization criterion. The simulation results verify the effectiveness of the proposed controller used on both fractional-order and integer-order systems. The systems controlled by the proposed algorithm

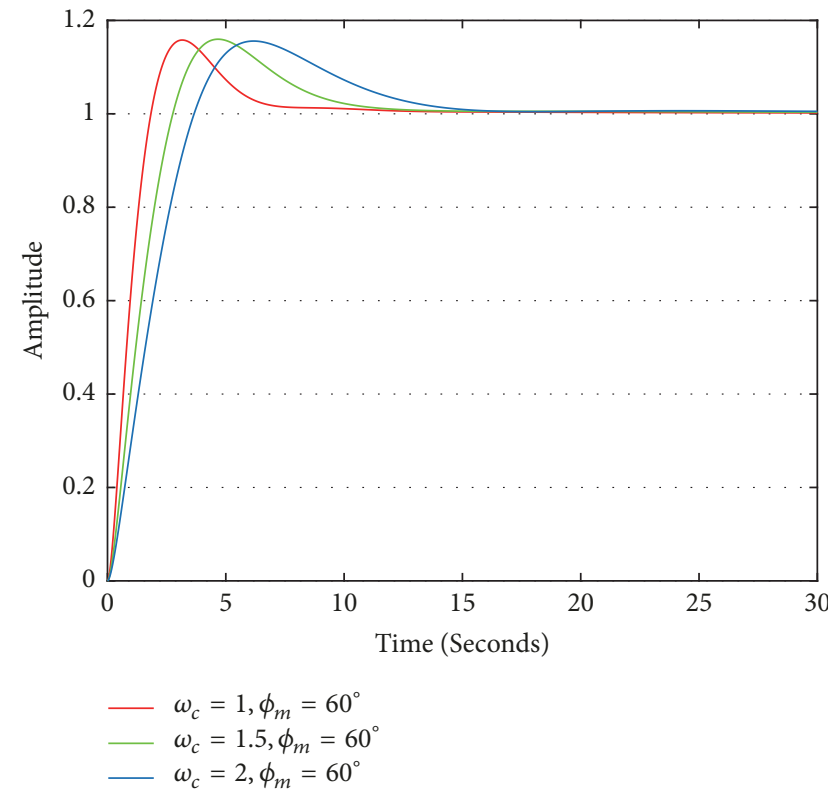

FIGURE 21: Step response of $P_{3}(s)$ with the same $\phi_{m}$.

achieve better robustness and transient control performance compared with other controllers.

\section{Conclusion}

In this paper, a novel tuning methodology of robust fractional-order PID controller is proposed. The controlled system output can be shaped by varying reference model parameters according to different control performance requirements. The phase curve can be flat within a certain frequency limit. Therefore, the system has the desirable characteristic of being robust to gain variations. Robustness analysis which supports the robust tuning specification is also carried out. The proposed fractional-order PID controller does not have any restriction on the controlled plant. So it can be widely applied on both integer-order and fractional-order systems. 


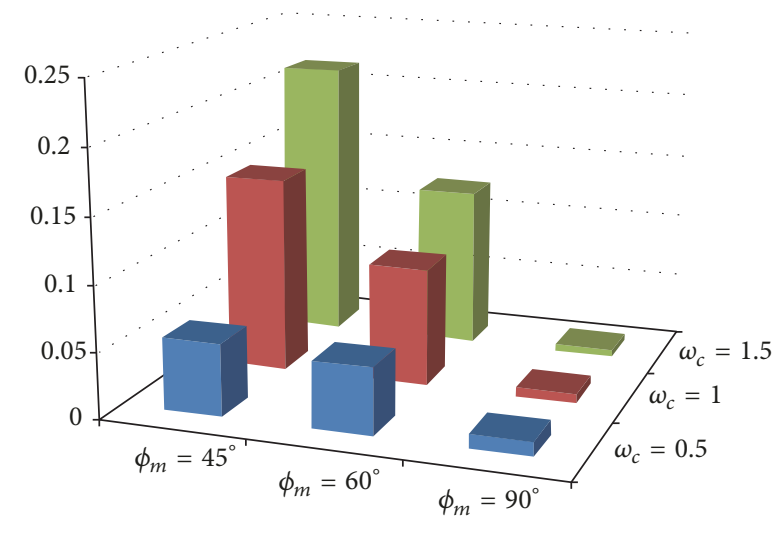

FIgURE 22: ITAE indices values of $P_{3}(s)$.

Three design examples including different kinds of controlled plants are presented to verify the effectiveness of the proposed algorithm.

\section{Conflicts of Interest}

The authors declare that they have no conflicts of interest.

\section{Acknowledgments}

This work is supported by the Fundamental Research Funds for the Central Universities of China (under Grant nos. G2018KY0305, G2018KY0302, and 3102017OQD069) and the National Natural Science Foundation of China (NSFC) (under Grant no. 61703335).

\section{References}

[1] D. Xue, Fractional-order Control Systems: Fundamentals and Numerical Implementations, De Gruyter, Berlin, 2017.

[2] C. A. Monje, B. M. Vinagre, V. Feliu, and Y. Chen, "Tuning and auto-tuning of fractional order controllers for industry applications," Control Engineering Practice, vol. 16, no. 7, pp. 798-812, 2008.

[3] L. Liu, S. Tian, D. Xue, T. Zhang, and Y. Chen, "Continuous fractional-order Zero Phase Error Tracking Control," ISA Transactions, vol. 75, pp. 226-235, 2018.

[4] L. Liu, F. Pan, and D. Xue, "Variable-order fuzzy fractional PID controller," ISA Transactions, vol. 55, pp. 227-233, 2015.

[5] A. Lassoued and O. Boubaker, "Dynamic analysis and circuit design of a novel hyperchaotic system with fractional-order terms," Complexity, vol. 2017, Article ID 3273408, 10 pages, 2017.

[6] S. Zhang, Y. Yu, and H. Wang, "Mittag-Leffler stability of fractional-order Hopfield neural networks," Nonlinear Analysis: Hybrid Systems, vol. 16, pp. 104-121, 2015.

[7] S. Zhang, Y. Yu, and Q. Wang, "Stability analysis of fractionalorder Hopfield neural networks with discontinuous activation functions," Neurocomputing, vol. 171, pp. 1075-1084, 2016.

[8] R. L. Magin, "Fractional calculus in bioengineering, part 3," Critical Reviews in Biomedical Engineering, vol. 32, no. 1, pp. 1104, 2004

[9] T. J. Freeborn, "A survey of fractional-order circuit models for biology and biomedicine," IEEE Journal on Emerging and
Selected Topics in Circuits and Systems, vol. 3, no. 3, pp. 416-424, 2013.

[10] F. Ge, Y. Chen, and C. Kou, "On the regional gradient observability of time fractional diffusion processes," Automatica, vol. 74, pp. 1-9, 2016.

[11] K. J. Strm and T. Hgglund, PID controllers: Theory, Design and Tuning, 1995.

[12] I. Podlubny, "Fractional-order systems and $\mathrm{PI}^{\lambda} \mathrm{D}^{\mu}$-controllers," IEEE Transactions on Automatic Control, vol. 44, no. 1, pp. 208214, 1999.

[13] I. Petras, "Fractional-order feedback control of a DC motor," Journal of Electrical Engineering, vol. 60, no. 3, pp. 117-128, 2009.

[14] M. Zamani, M. Karimi-Ghartemani, N. Sadati, and M. Parniani, "Design of a fractional order PID controller for an AVR using particle swarm optimization," Control Engineering Practice, vol. 17, no. 12, pp. 1380-1387, 2009.

[15] B. Vinagre, I. Podlubny, L. Dorcak, and V. Feliu, "On fractional PID controllers: A frequency domain approach," in Proceedings of the IFAC Workshop on Digital Control Past, 2000.

[16] S. E. Hamamci, "An algorithm for stabilization of fractionalorder time delay systems using fractional-order PID controllers," Institute of Electrical and Electronics Engineers Transactions on Automatic Control, vol. 52, no. 10, pp. 1964-1968, 2007.

[17] H. U. Hai-Bo and Y. R. Huang, "Self-tuning fractional order PID based on hybrid PSO neural networks," in Microelectronics \& Computer, 2010.

[18] A. Oustaloup, J. Sabatier, P. Lanusse et al., "An overview of the CRONE approach in system analysis, modeling and identification, observation and control," IFAC Proceedings Volumes, vol. 41, no. 2, pp. 14254-14265, 2008.

[19] Y. Chen and K. L. Moore, "Relay feedback tuning of robust PID controllers with iso-damping property," IEEE Transactions on Systems, Man, and Cybernetics, Part B: Cybernetics, vol. 35, no. 1, pp. 23-31, 2005.

[20] H. S. Li, Y. Luo, and Y. Q. Chen, "A fractional order proportional and derivative (FOPD) motion controller: tuning rule and experiments," IEEE Transactions on Control Systems Technology, vol. 18, no. 2, pp. 516-520, 2010.

[21] Y. Luo, Y. Q. Chen, C. Y. Wang, and Y. G. Pi, “Tuning fractional order proportional integral controllers for fractional order systems," Journal of Process Control, vol. 20, no. 7, pp. 823-831, 2010.

[22] Y. Chen, H. Dou, B. M. Vinagre, and C. A. Monje, "A robust tuning method for fractional order PI controllers," IFAC Proceedings Volumes, vol. 39, no. 11, pp. 22-27, 2006.

[23] L. Liu, S. Zhang, D. Xue, and Y. Chen, "General robustness analysis and robust fractional-order PD controller design for fractional-order plants," IET Control Theory Application, 2018.

[24] V. Feliu-Batlle, R. Rivas Pérez, F. J. Castillo García, and L. Sanchez Rodriguez, "Smith predictor based robust fractional order control: application to water distribution in a main irrigation canal pool," Journal of Process Control, vol. 19, no. 3, pp. 506-519, 2009.

[25] V. Feliu-Batlle, "Robust isophase margin control of oscillatory systems with large uncertainties in their parameters: a fractional-order control approach," International Journal of Robust and Nonlinear Control, vol. 27, no. 12, pp. 2145-2164, 2017.

[26] C. Yeroglu and N. Tan, "Note on fractional-order proportionalintegral-differential controller design," IET Control Theory \& Applications, vol. 5, no. 17, pp. 1978-1989, 2011. 
[27] V. Kumar, K. P. Rana, and P. Mishra, "Robust speed control of hybrid electric vehicle using fractional order fuzzy PD and PI controllers in cascade control loop," Journal of The Franklin Institute, vol. 353, no. 8, pp. 1713-1741, 2016.

[28] H. W. Bode, Network Analysis and Feedback Amplifier Design, Van Nostrand, 1945.

[29] A. Banos, J. n. Cervera, P. Lanusse, and J. Sabatier, "Bode optimal loop shaping with CRONE compensator," Journal of Vibration and Control, vol. 17, no. 13, pp. 1964-1974, 2011.

[30] R. Azarmi, M. Tavakoli-Kakhki, A. K. Sedigh, and A. Fatehi, "Robust Fractional Order PI Controller Tuning Based on Bode's Ideal Transfer Function," IFAC-PapersOnLine, vol. 49, no. 9, pp. 158-163, 2016.

[31] K. Amoura, R. Mansouri, M. Bettayeb, and U. M. Al-Saggaf, "Closed-loop step response for tuning PID-fractional-orderfilter controllers," ISA Transactions, vol. 64, pp. 247-257, 2016.

[32] R. Malti, X. Moreau, F. Khemane, and A. Oustaloup, "Stability and resonance conditions of elementary fractional transfer functions," Automatica, vol. 47, no. 11, pp. 2462-2467, 2011.

[33] R. S. Barbosa, J. A. T. Machado, and I. M. Ferreira, "Tuning of PID controllers based on bode's ideal transfer function," Nonlinear Dynamics, vol. 38, no. 1-4, pp. 305-321, 2004.

[34] A. Oustaloup, P. Melchior, P. Lanusse, O. Cois, and F. Dancla, The CRONE toolbox for MATLAB, 2000.

[35] M. A. Luersen and R. le Riche, "Globalized nelder-mead method for engineering optimization," Computers \& Structures, vol. 82, no. 23-26, pp. 2251-2260, 2004.

[36] R. Azarmi, A. K. Sedigh, M. Tavakoli-Kakhki, and A. Fatehi, "Design and implementation of smith predictor based fractional order PID controller on MIMO flow-level plant," in Proceedings of the 23rd Iranian Conference on Electrical Engineering, ICEE 2015, pp. 858-863, Iran, May 2015.

[37] F. Padula, R. Vilanova, and A. Visioli, " $H_{\infty}$ optimization-based fractional-order PID controllers design," International Journal of Robust and Nonlinear Control, vol. 24, no. 17, pp. 3009-3026, 2014.

[38] D. Wang and X. Gao, " $\mathrm{H}_{\infty}$ design with fractional-order $\mathrm{PD}^{\mu}$ controllers," Automatica, vol. 48, no. 5, pp. 974-977, 2012. 


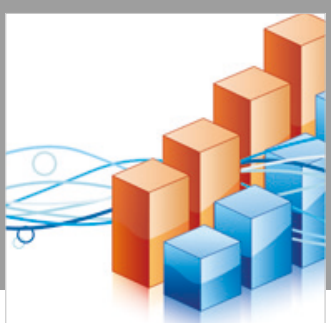

Advances in

Operations Research

\section{-n-m}
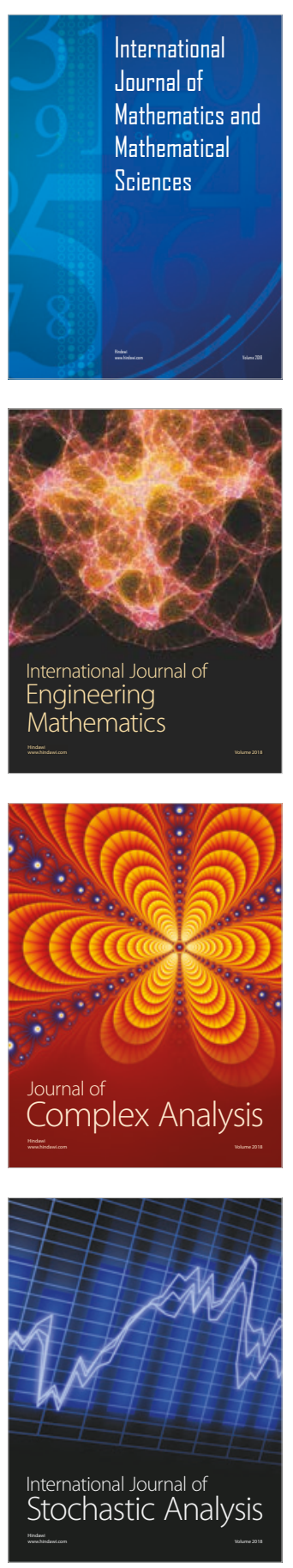
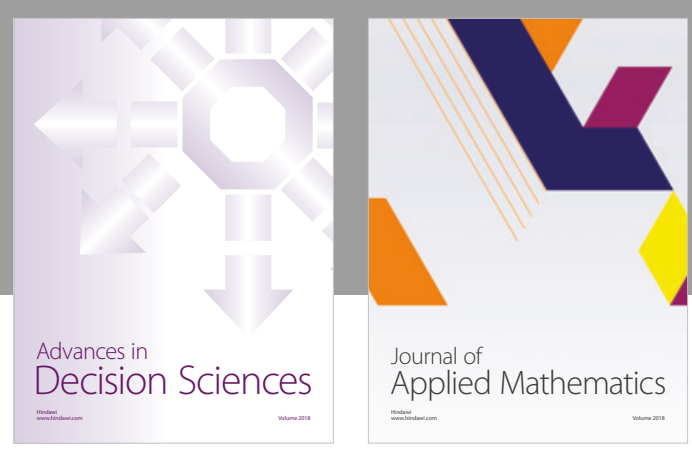

Journal of

Applied Mathematics
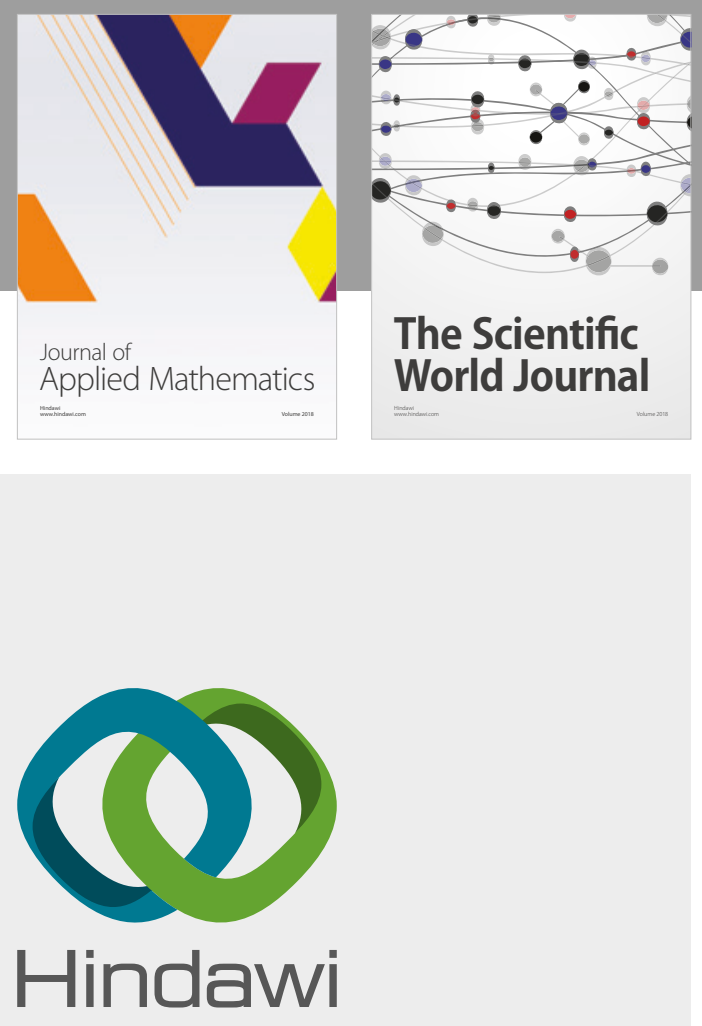

Submit your manuscripts at

www.hindawi.com

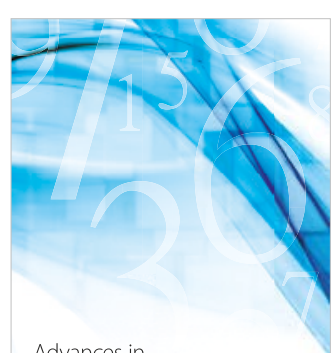

Advances in
Numerical Analysis
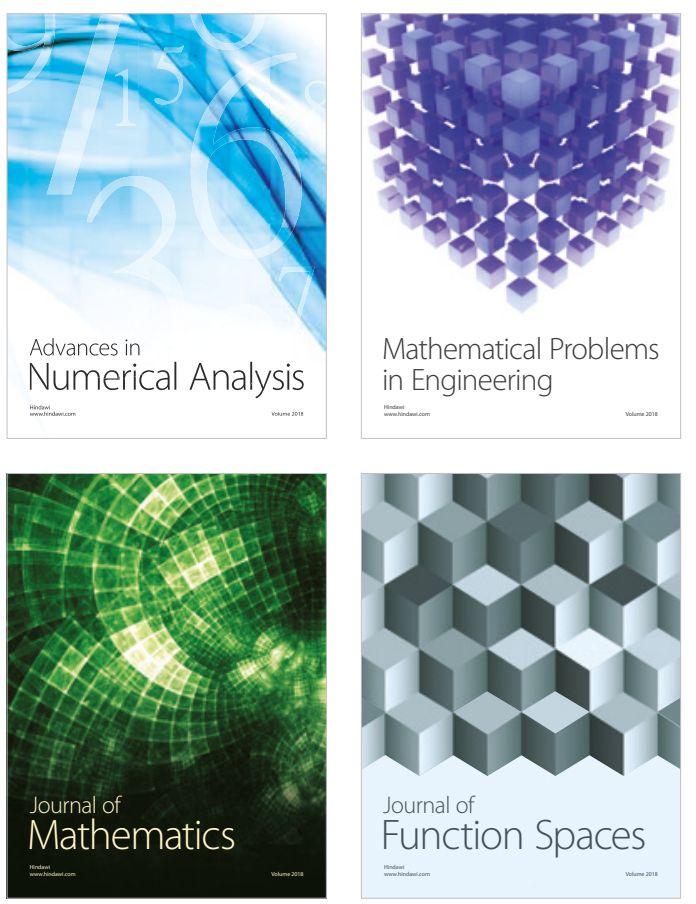

Mathematical Problems in Engineering

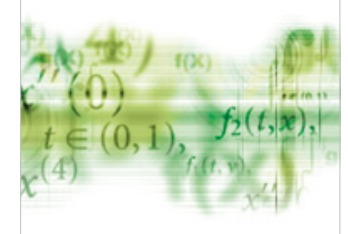

International Journal of

Differential Equations

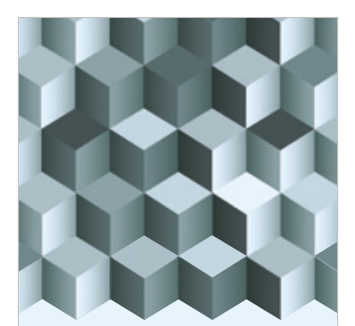

Journal of

Function Spaces
The Scientific

World Journal

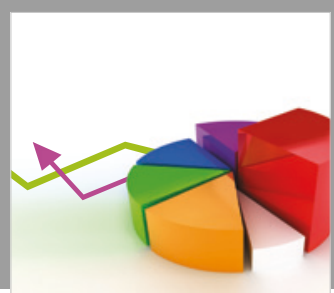

Journal of

Probability and Statistics
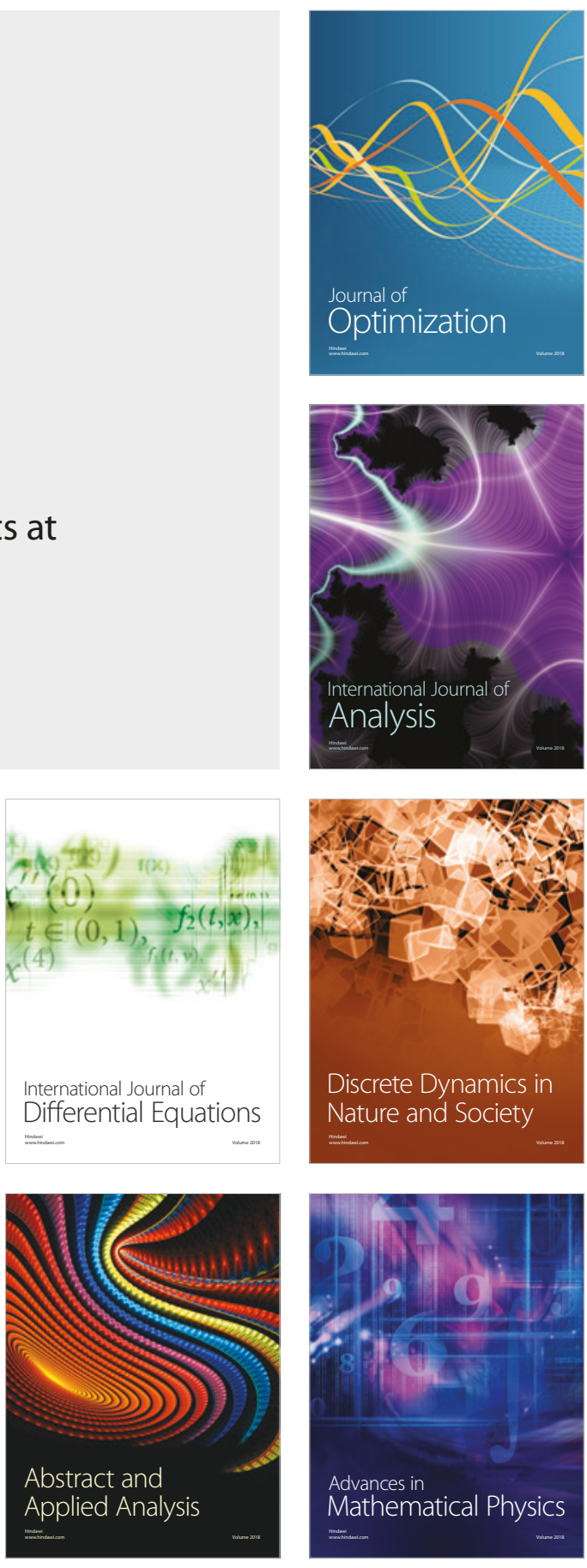